\title{
Single administration of fluoxetine improves memory function without neuroprotection after cardiac arrest/cardiopulmonary resuscitation in mice
}

\author{
Noriko TAGUCHI', Shin NAKAYAMA², MakotoTANAKA² \\ 1 Tsukuba University Hospital / Mito Kyodo General Hospital, Dept. of Anesthesiology, Mito, Japan \\ 2 University of Tsukuba, Dept. of Anesthesiology, Tsukuba, Japan
}

\section{BACKGROUND \& GOAL OF STUDY}

Several clinical studies have indicated that serotonin re-uptake inhibitor (SSRI) administration after acute ischemic stroke can improve clinical recovery. Fluoxetine (FLX: one of the SSRIs) has several mechanisms which contribute to relieve the ischemic brain damage. We have previously reported that FLX had neuroprotective effects on day 3 after cardiac arrest and cardiopulmonary resuscitation (CA/CPR) in mice (1). However, the neuronal loss after global ischemia is suspected to continue up to 14 days after insults (2). The goal of this study is to evaluate the neuroprotective effect of FLX for a longer observation period.

\section{MATERIALS \& METHODS}

\section{Animals}

- Male C57BL/6 mice

Age; 8-10 weeks

Weight; $22-26 \mathrm{~g}$

$\mathrm{n}=12$ (each group at Protocol 1), n=24 (each group at Protocol 2) Groups

- FLX group: Group F ( FLX $10 \mathrm{mg} / \mathrm{kg}$, dissolved in normal saline)

- Control group: Group C ( normal saline)

$>$ Intra-peritoneal injection was completed $30 \mathrm{~min}$ after recovery of spontaneous circulation to each experiment.

Protocol 1

- CA (7 min)/ CPR was induced as previously described (1).

$>$ Behavioral test; Six and seven days after CA/CPR

$>$ Histological evaluation; Seven days after CA/CPR

Protocol 2

- CA/CPR methods were same as protocol 1

$>$ Behavioral test; Six, seven and fourteen days after CA/CPR

$>$ Histological evaluation; Fourteen days after CA/CPR

Measurement

- Immunostaining for histological evaluation

$>$ Protocol 1: Surviving neuron (Neu-N), microglia (IBA-1)

$>$ Protocol 2: Survival neuron (Neu-N), immature neuron (Doublecortin)

- Passive avoidance test was conducted as behavioral test

Analysis

- Unpaired t test or Mann-Whitney U test (behavioral test analysis).

- Chi-squared test (survival analysis)

- The $P$ value less than 0.05 was considered to be statistically significant.

\section{RESULTS}

NeuN positive cells
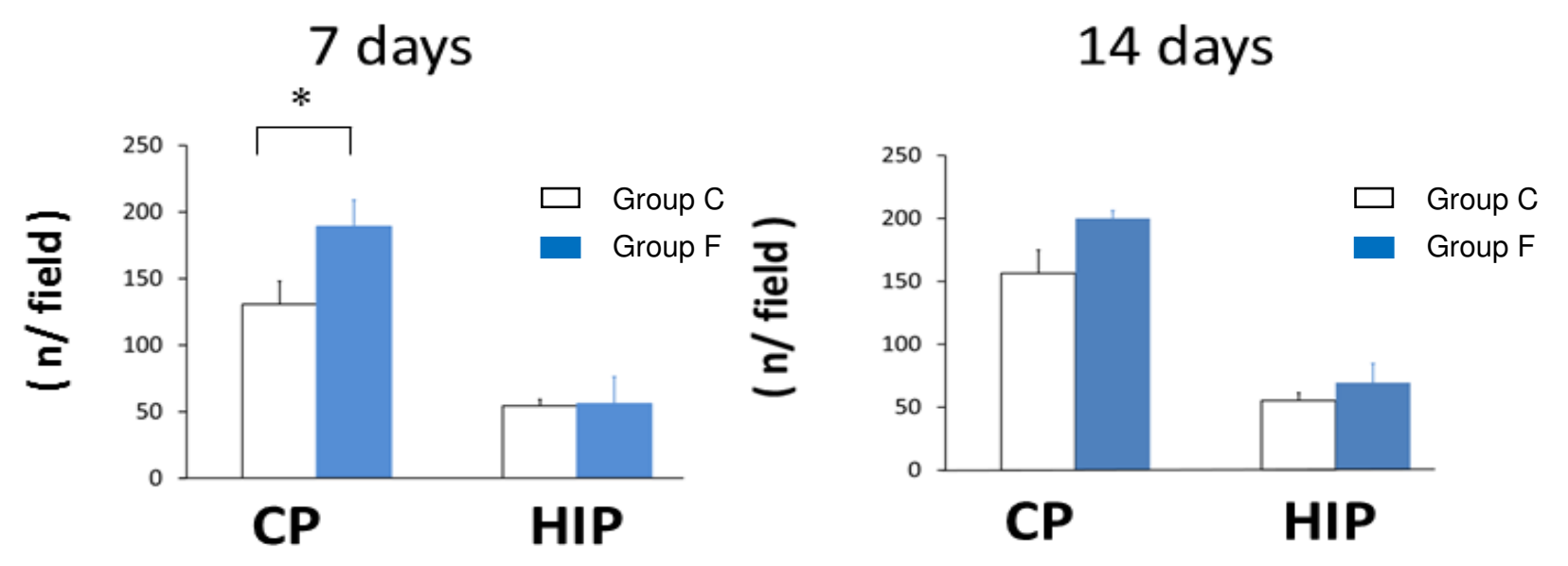

CP: Caudate Putamen HIP: Hippocampus
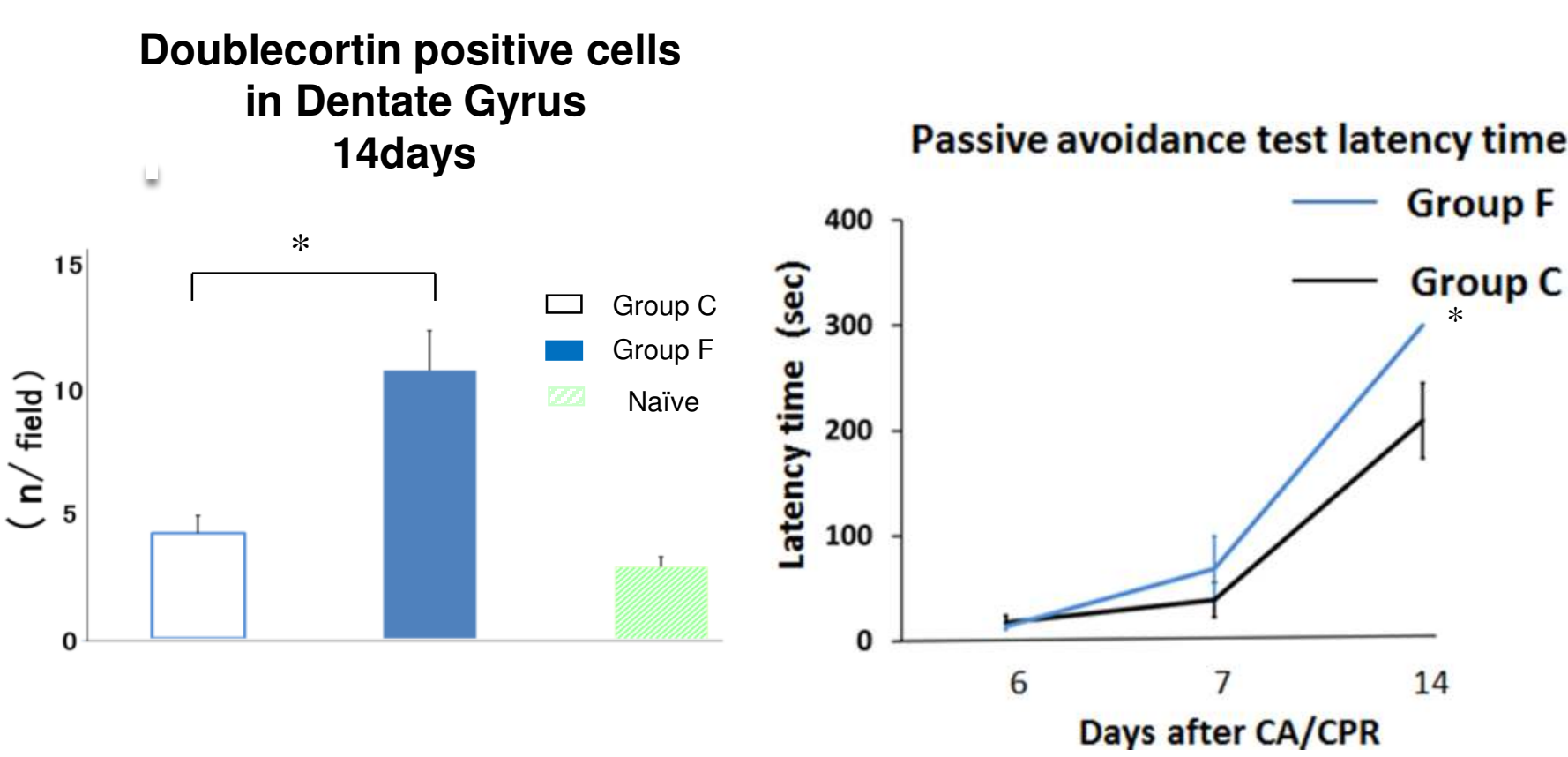

Fourteen days after CA/CPR, the neuroprotective effect of fluoxetine was disappeared in number of surviving neurons.

- Fluoxetine significantly increased the number of immature neurons in dentate gyrus and improved memory function on 14 days after CA/CPR

- In this experiment, the causal relationship between neurogenesis and the improvement of memory function was not clear.

\section{CONCLUSIONS}

We conclude that fluoxetine improves memory function on 14 days after global brain ischemia. - Our results may suggest that the functional recovery induced by fluoxetine is attributed to the other factors than the simple neuronal protection. 